\title{
Algorithms for the recovery of Kohlrausch parameters from viscoelastic stress-strain data
}

\author{
Saiful A. Husain* $\quad$ R. S. Anderssen ${ }^{\dagger}$
}

(Received 29 January 2004, revised 29 July 2005)

\begin{abstract}
The Boltzmann model of linear viscoelasticity is an appropriate model for materials that simultaneously exhibit viscous and elastic behaviour, such as synthetic and natural polymers. The nature of the viscoelasticity is encapsulated in terms of the structure of its kernel function (the relaxation modulus) $G(t)$. Husain and Anderssen (2005) proposed a procedure for approximating $G(t)$, based on taking moments of the Boltzmann equation with $G(t)$ the sum of Kohlrausch functions. The shortcoming of this proposal is the need to evaluate the moments on the half-interval $[0, \infty)$. We propose a method which takes the strain-rates to have a polynomial form and $G(t)$ to be a sum
\end{abstract}

${ }^{*}$ CMA, Mathematical Sciences Institute, Australian National University, Canberra, ACT 0200 Australia; Department of Mathematics, Faculty of Science, Universiti Brunei Darussalam, Gadong, BE 1410 BruneI. mailto:Saiful@fos.ubd.edu.bn

${ }^{\dagger}$ CSIRO, Mathematical and Information Sciences, GPO Box 664, Canberra, ACT 2601, Australia. mailto:Bob.Anderssen@csiro.au

See http://anziamj.austms.org.au/V46/CTAC2004/Husa for this article, (C) Austral. Mathematical Soc. 2005. Published September 22, 2005. ISSN 1446-8735 
of Kohlrausch functions. It yields methods for the direct estimation of the parameters in Kohlrausch models for $G(t)$.

\section{Contents}

1 Introduction

2 Preliminaries and notation $\quad \mathbf{C 9 3 9}$

2.1 Kohlrausch functions . . . . . . . . . . . . . . . . . C939

2.2 Incomplete Gamma function . . . . . . . . . . . . . . C C940

2.3 Feng Qi inequality . . . . . . . . . . . . . C940

2.4 Inequality for incomplete Gamma function ratios . . . . . C940

2.5 The Boltzmann model for strain-rates defined on a finite interval . . . . . . . . . . . . . . .

3 The stress and derivative of stress for polynomial strainrates

C942

4 The moments of the stress for polynomial strain-rates

C944

5 Analytic expression for stress for the single Kohlrausch C946 5.1 Recovery of Kohlrausch parameters: single Kohlrausch . . C948

A Monotonicity of $F_{1}\left(\tau_{1}, \beta_{1}\right)$

C951

References

C954 


\section{Introduction}

The Boltzmann model of linear viscoelasticity is

$$
\sigma(t)=\int_{0}^{t} G(t-\tau) \dot{\gamma}(\tau) d \tau
$$

where

$$
\begin{aligned}
\sigma(t) & :=\text { stress at time } t \\
G(t) & :=\text { the relaxation modulus, } \\
\gamma(t) & :=\text { the strain, } \\
\dot{\gamma}(t) & :=d \gamma / d t, \text { the strain rate, } \\
\tau & :=\text { the relaxation time. }
\end{aligned}
$$

The nature of the material under investigation (for example, synthetic and biopolymers, bone, collagen) is encapsulated in the form of the relaxation modulus $G(t)$ which, in order to guarantee sensible physics, is normally chosen to be completely monotone. The traditional strategy for guaranteeing that any approximation to $G(t)$ is a complete monotone function, has been to solve the oscillatory shear rheometry equations with

$$
G(t)=\int_{0}^{\infty} \exp (-t / \tau) \frac{H(\tau)}{\tau} d \tau, \quad H(\tau) \geq 0,
$$

where $H(\tau)$ denotes the corresponding relaxation time spectrum. With respect to the measured storage and loss modulus data, the algorithm solves the corresponding equations for $H(\tau)$ and then substitutes this estimate into the relaxation spectrum equation for $G(t)$ (Davies and Anderssen [7]).

Husain and Anderssen [11] recently showed how moments of the Boltzmann model of linear viscoelasticity can be evaluated analytically when the kernel (relaxation modulus) $G(t)$ is approximated as a sum of Kohlrausch 
functions. These results were then used to derive simple hybrid analyticnumerical algorithms for the estimation of the unknown parameters in the chosen Kohlrausch function model. The immediate appeal of the resulting algorithms related to: their ease of implementation; the evaluation of the required moments can be performed in a stable manner; and one only needs as many moments of the stress (and corresponding moments of the strain-rate) as the number of parameters in the Kohlrausch function approximation.

The advantage of this method is that it applies for a more or less arbitrary choice for the strain-rate, and leads to a quite simple algorithm for the recovery of the Kohlrausch parameters. Its potential disadvantage is that the moments must be evaluated on the half-interval $[0, \infty)$. This limits its applicability because, in an experiment, the stress is only available as measurements at a discrete set of points. Either, for a given strain-rate, an experiment must be performed until the measured stress has effectively reached zero, or a specific strain-rate must be chosen which guarantees that the stress will have essentially reached zero before the measurement of the stress is halted.

In this paper, alternative methods are proposed which circumvents the need to evaluate moments on the half interval $[0, \infty)$ by restricting attention to a special sub-class of strain-rates which have a polynomial structure and are defined on some compact interval $[0, a]$. From a practical rheological perspective, the advantages of this alternative approach include:

1. such strain-rates are important as they have the type of form suitable for an actual rheometry experiment;

2. they yield an analytic model for the stress, to be fitted to the measured stress to yield a more appropriate assessment of the actual stress;

3. they yield analytic models for the moments that can be exploited to directly recover an estimate of the Kohlrausch parameters. 


\section{Preliminaries and notation}

The Boltzmann equation (1) models how the stress $\sigma(t)$, at time $t$, depends on the earlier history of the strain $\gamma(t)$ and strain rate $\dot{\gamma}(\tau)[6]$. In the formulation of a Boltzmann model, the key consideration is the choice of the relaxation modulus $G(t)$. The regularity imposed on $G(t)$ is such that it must have a fading memory. A popular choice $[5,8]$ is to assume that $G(t)$ is completely monotone $[9,14]$.

The Kohlrausch function is often referred to as the Kohlrausch-WilliamsWatts function to recognise the important contribution of Graham Williams and David Watts in 1970 [15] in identifying the role that this function could play in modelling various forms of physical, chemical and polymeric relaxation processes. It also recognises the subsequent huge impact of that and other related papers. A review of the extent and significance of this impact, as well as the original papers by Williams and colleagues, is found in Williams [16].

\subsection{Kohlrausch functions}

One of the possible choices for a completely monotone $G(t)$ is the Kohlrausch (stretched exponential; Williams-Watts) function [2, 3, 9, 10]

$$
K_{\tau_{\ell}, \beta_{\ell}}(t)=\exp \left(-\left(t / \tau_{\ell}\right)^{\beta_{\ell}}\right), \quad 0<\beta<1, \quad \ell=1,2, \ldots, L .
$$

The Kohlrausch function also proved to be more appropriate in modelling the associated relaxation and decay processes than the standard exponential function. Further details about the key properties of the Kohlrausch functions and the merits of using the Kohlrausch function can be found in [4]. Kohlrausch in 1854 [13] proposed using the above expression (2) as a relaxation function for dynamical processes in materials in the study of creep in electric displacements. In order to allow for a more general choice of the 
relaxation modulus $G(t)$, we assume that $G(t)$ is a sum of the Kohlrausch functions:

$$
G_{L}(t)=\sum_{\ell=1}^{L} k_{\ell} K_{\tau_{\ell}, \beta_{\ell}}(t),
$$

where the $\beta_{\ell}$ and the $\tau_{\ell}$ correspond to the Kohlrausch exponents and relaxation times.

\subsection{Incomplete Gamma function}

Let $\Phi_{x}(s)$ denote the lower incomplete gamma function [1]:

$$
\Phi_{x}(s)=\int_{0}^{x} t^{s-1} \exp (-t) d t
$$

\subsection{Feng Qi inequality}

For the subsequent analysis, the following inequality of Feng Qi [12] is required.

Let $g(t)$ be a locally integrable positive function on the interval between $x$ and $y, x, y \in \mathbb{R}$, such that $\exp (t) g(t)$ is decreasing, then

$$
\frac{\Phi_{x}(s)}{\Phi_{x}(r)} \geq \frac{\int_{0}^{x} t^{s-1} g(t) d t}{\int_{0}^{x} t^{r-1} g(t) d t}
$$

holds for $s>r>0$ and $x>0$. If $\exp (t) g(t)$ is increasing, then the above inequality reverses.

\subsection{Inequality for incomplete Gamma function ratios}

For the subsequent analysis, the following proposition is required. 
Proposition 1 For fixed $\tau_{1}, n \in \mathbb{R}, n>1$,

$$
\frac{\Phi_{T\left(\beta_{1}, \tau_{1}\right)}\left(\frac{n}{\beta_{1}}\right)}{T\left(\beta_{1}, \tau_{1}\right)^{n / \beta_{1}-1} \Phi_{T\left(\beta_{1}, \tau_{1}\right)}(1)}<1 .
$$

Proof: One exploits the Feng Qi Inequality given in the Subsection 2.3. For $s=n / \beta_{1}$ and $r=1, g(t)=t$ is an integrable positive function on the interval between 0 and $T\left(\beta_{1}, \tau_{1}\right)$ because

$$
\int_{0}^{T\left(\beta_{1}, \tau_{1}\right)} t d t=T^{2}\left(\beta_{1}, \tau_{1}\right) / 2<\infty,
$$

and, consequently, $\exp (t) g(t)=t \exp (t)$ is increasing. For this choice, the inequality yields

$$
\frac{\Phi_{T\left(\beta_{1}, \tau_{1}\right)}\left(\frac{n}{\beta_{1}}\right)}{\Phi_{T\left(\beta_{1}, \tau_{1}\right)}(1)} \leq \frac{2 \beta_{1}}{\left(\beta_{1}+n\right)} T\left(\beta_{1}, \tau_{1}\right)^{n / \beta_{1}-1} .
$$

Finally, dividing both sides of inequality (8) by $T\left(\beta_{1}, \tau_{1}\right)^{n / \beta_{1}-1}$ yields

$$
\frac{\Phi_{T\left(\beta_{1}, \tau_{1}\right)}\left(\frac{n}{\beta_{1}}\right)}{T\left(\beta_{1}, \tau_{1}\right)^{n / \beta_{1}-1} \Phi_{T\left(\beta_{1}, \tau_{1}\right)}(1)} \leq \frac{2 \beta_{1}}{\left(\beta_{1}+n\right)} .
$$

Since $0<\beta_{1}<1$, then $1 / \beta_{1}>1$. Hence

$$
\frac{2 \beta_{1}}{\left(\beta_{1}+n\right)}=\frac{2}{\left(1+n / \beta_{1}\right)}<1
$$

Therefore, one obtains the inequality (6).

\subsection{The Boltzmann model for strain-rates defined on a finite interval}

Without loss of generality, assume that the finite interval is the unit interval $[0,1]$, and that $\gamma(t)=\varphi(t) H(t-1)$, where $H(t-1)$ denotes the Heaviside 
step function. It follows that $\dot{\gamma}(t)=\dot{\varphi}(t) H(t-1)+\varphi(t) \delta(t-1)$, where $\delta(t-1)$ denotes the Dirac Delta function. Substitution of this result into equation (1) yields

$$
\sigma(t)=\int_{0}^{t} G(t-\tau)\{\dot{\varphi}(\tau) H(\tau-1)+\varphi(\tau) \delta(\tau-1)\} d \tau .
$$

Some algebraic manipulation, using the properties of $H(\tau-1)$ and $\delta(\tau-1)$, of equation (11) yields

$$
\sigma(t)= \begin{cases}\int_{0}^{t} G(t-\tau) \dot{\varphi}(\tau) d \tau, & 0 \leq t<1 \\ \int_{0}^{1} G(t-\tau) \dot{\varphi}(\tau) d \tau+G(t-1) \varphi(1), & t \geq 1\end{cases}
$$

If $\varphi(1)=0$, equation (12) becomes

$$
\sigma(t)= \begin{cases}\int_{0}^{t} G(t-\tau) \dot{\varphi}(\tau) d \tau, & 0 \leq t<1 \\ \int_{0}^{1} G(t-\tau) \dot{\varphi}(\tau) d \tau, & t \geq 1\end{cases}
$$

\section{The stress and derivative of stress for polynomial strain-rates}

Proposition 2 For $\dot{\gamma}(t)=c t^{p}$,

$$
\begin{aligned}
\sigma_{L}(t) & =c \sum_{\ell=1}^{L} k_{\ell} \int_{0}^{t} K_{\tau_{\ell}, \beta_{\ell}}(t-\tau) \tau^{p} d \tau \\
& =c \sum_{\ell=1}^{L} \frac{k_{\ell} \tau_{\ell}}{\beta_{\ell}}\left[\sum_{i=0}^{p} \tilde{C}_{i}^{(\ell)} \Phi_{T\left(\beta_{\ell}, \tau_{\ell}\right)}\left(\frac{p-i+1}{\beta_{\ell}}\right)\right],
\end{aligned}
$$

where

$$
\tilde{C}_{i}^{(\ell)}=\left(\begin{array}{c}
p \\
i
\end{array}\right) t^{i}\left(-\tau_{\ell}\right)^{p-i}
$$


Proof: Substituting equation (3) into equation (1) gives

$$
\sigma_{L}(t)=\sum_{\ell=1}^{L} k_{\ell} \int_{0}^{t} K_{\tau_{\ell}, \beta_{\ell}}(t-\tau) \dot{\gamma}(\tau) d \tau
$$

Substitution of $\dot{\gamma}(t)=c t^{p}$ into equation (17) yields equation (14). The substitution $\phi=\left((t-\tau) / \tau_{\ell}\right)^{\beta_{\ell}}$ into equation (17) yields

$$
\sigma_{L}(t)=c \sum_{\ell=1}^{L} \frac{k_{\ell} \tau_{\ell}}{\beta_{\ell}}\left[\sum_{i=0}^{p} \tilde{C}_{i}^{(\ell)} \int_{0}^{\left(t / \tau_{\ell}\right)^{\beta_{\ell}}} \phi^{(p-i+1) / \beta_{\ell}-1} \exp (-\phi) d \phi\right],
$$

where $\tilde{C}_{i}^{(\ell)}=\left(\begin{array}{c}p \\ i\end{array}\right) t^{i}\left(-\tau_{\ell}\right)^{p-i}$.

One now uses

$$
\int_{0}^{T\left(\beta_{\ell}, \tau_{\ell}\right)} \phi^{(p-i+1) / \beta_{\ell}-1} \exp (-\phi) d \phi=\Phi_{T\left(\beta_{\ell}, \tau_{\ell}\right)}\left(\frac{p-i+1}{\beta_{\ell}}\right),
$$

where $T\left(\beta_{\ell}, \tau_{\ell}\right)=\left(t / \tau_{\ell}\right)^{\beta_{\ell}}$, to obtain equation (17).

Proposition 3 For $\dot{\gamma}(t)=c t^{p}$, the derivative of the stress

$$
\dot{\sigma}_{L}(t)=\sum_{\ell=1}^{L} k_{\ell} c\left[t^{p}-\sum_{i=0}^{p} \tilde{C}_{i}^{(\ell)} \Phi_{T\left(\beta_{\ell}, \tau_{\ell}\right)}\left(\frac{p-i+\beta_{\ell}}{\beta_{\ell}}\right)\right] .
$$

Proof: Differentiating $\sigma_{L}(t)$, that is, equation (17), with respect to time $t$, yields

$$
\dot{\sigma}_{L}(t)=\sum_{\ell=1}^{L} k_{\ell}\left(\dot{\gamma}(t)-\frac{\beta_{\ell}}{\tau_{\ell}^{\beta_{\ell}}} \int_{0}^{t}(t-\tau)^{\beta_{\ell}-1} \exp \left(-\left((t-\tau) / \tau_{\ell}\right)^{\beta_{\ell}}\right) \dot{\gamma}(\tau) d \tau\right) .
$$


To evaluate

$$
I=\int_{0}^{t}(t-\tau)^{\beta_{\ell}-1} \exp \left(-\left((t-\tau) / \tau_{\ell}\right)^{\beta_{\ell}}\right) \dot{\gamma}(\tau) d \tau
$$

one uses the substitution $\phi=\left((t-\tau) / \tau_{\ell}\right)^{\beta_{\ell}}$ and $\dot{\gamma}(t)=c t^{p}$ into equation (21) to obtain

$$
\begin{aligned}
I & =c \frac{\tau_{\ell}^{\beta_{\ell}}}{\beta_{\ell}} \sum_{i=0}^{p} \tilde{C}_{i}^{(\ell)} \int_{0}^{\left(t / \tau_{\ell}\right)^{\beta_{\ell}}} \phi^{\left(p-i+\beta_{\ell}\right) / \beta_{\ell}-1} \exp (-\phi) d \phi \\
& =c \frac{\tau_{\ell}^{\beta_{\ell}}}{\beta_{\ell}} \sum_{i=0}^{p} \tilde{C}_{i}^{(\ell)} \Phi_{T\left(\beta_{\ell}, \tau_{\ell}\right)}\left(\frac{p-i+\beta_{\ell}}{\beta_{\ell}}\right) .
\end{aligned}
$$

Finally, the substitution of equation (23) into equation (20) yields equation (19).

\section{The moments of the stress for polynomial strain-rates}

For polynomial strain-rates, finite moments of the stress can be evaluated analytically. For fixed $0 \leq \bar{T}<\infty$, the moments of the stress $\sigma_{L}(t)$ are

$$
M_{m}^{(L)}=\int_{0}^{\bar{T}} t^{m} \sigma_{L}(t) d t=\sum_{\ell=1}^{L} k_{\ell} \int_{0}^{\bar{T}} t^{m}\left[\int_{0}^{t} K_{\tau_{\ell}, \beta_{\ell}}(t-\tau) \dot{\gamma}(\tau) d \tau\right] d t .
$$

Proposition 4 For a strain-rate $\dot{\gamma}(t)=c t^{p}$, where $p$ is a positive integer and $c$ is a positive constant,

$$
M_{m}^{(L)}=c \sum_{\ell=1}^{L} \frac{k_{\ell} \tau_{\ell}}{\beta_{\ell}} \sum_{i=0}^{m}\left(\begin{array}{c}
m \\
i
\end{array}\right) \tau_{\ell}^{i} \int_{0}^{\bar{T}} \Phi_{\overline{\bar{T}}}\left(\frac{i+1}{\beta_{\ell}}\right) \tau^{p+m-i} d \tau .
$$


Proof: To prove Proposition 4, one starts with equation (14). Multiplying expression (17) by the monomial $t^{m}$, where $m \geq 0$ is an integer, and then integrating the resulting expression from 0 to $\overline{\bar{T}}$ generates the moments of the stress given by (24). Changing the order of integration yields

$$
M_{m}^{(L)}=\sum_{\ell=1}^{L} k_{\ell} \int_{0}^{\bar{T}}\left[\int_{\tau}^{\bar{T}} t^{m} K_{\tau_{\ell}, \beta_{\ell}}(t-\tau) d t\right] \dot{\gamma}(\tau) d \tau .
$$

To evaluate

$$
I=\int_{\tau}^{\bar{T}} t^{m} K_{\tau_{\ell}, \beta_{\ell}}(t-\tau) d t
$$

use the substitution $u=t-\tau$ to obtain

$$
\begin{aligned}
I & =\int_{0}^{\bar{T}}(u+\tau)^{m} K_{\tau_{\ell}, \beta_{\ell}}(u) d u \\
& =\int_{0}^{\bar{T}} \sum_{i=0}^{m}\left(\begin{array}{c}
m \\
i
\end{array}\right) u^{i} \tau^{m-i} \exp \left(-\left(u / \tau_{\ell}\right)^{\beta_{\ell}}\right) d u .
\end{aligned}
$$

The substitution of $\phi=\left(u / \tau_{\ell}\right)^{\beta_{\ell}}$ into equation (27) yields

$$
I=\frac{\tau_{\ell}}{\beta_{\ell}} \sum_{i=0}^{m}\left(\begin{array}{c}
m \\
i
\end{array}\right) \tau_{\ell}^{i} \tau^{m-i} \int_{0}^{\left((\bar{T}-\tau) / \tau_{\ell}\right)^{\beta_{\ell}}} \phi^{(i+1) / \beta_{\ell}-1} \exp (-\phi) d \phi .
$$

One now uses

$$
\int_{0}^{\overline{\bar{T}}} \phi^{(i+1) / \beta_{\ell}-1} \exp (-\phi) d \phi=\Phi_{\overline{\bar{T}}}\left(\frac{i+1}{\beta_{\ell}}\right), \quad \overline{\bar{T}}=\left(\frac{\bar{T}-\tau}{\tau_{\ell}}\right)^{\beta_{\ell}},
$$

to obtain

$$
I=\frac{\tau_{\ell}}{\beta_{\ell}} \sum_{i=0}^{m}\left(\begin{array}{c}
m \\
i
\end{array}\right) \tau_{\ell}^{i} \tau^{m-i} \Phi_{\overline{\bar{T}}}\left(\frac{i+1}{\beta_{\ell}}\right) .
$$

Finally, the substitution of equation (30) into equation (26) yields

$$
M_{m}^{(L)}=\sum_{\ell=1}^{L} \frac{k_{\ell} \tau_{\ell}}{\beta_{\ell}} \sum_{i=0}^{m}\left(\begin{array}{c}
m \\
i
\end{array}\right) \tau_{\ell}^{i} \int_{0}^{\bar{T}} \Phi_{\overline{\bar{T}}}\left(\frac{i+1}{\beta_{\ell}}\right) \tau^{m-i} \dot{\gamma}(\tau) d \tau .
$$


Substitute the strain rate $\dot{\gamma}(t)=c t^{p}$ to yield equation (25). Using integration by parts, the following form of equation (25) can be derived (the derivation of the moments are omitted, since the construction of moments are not our mathematical focus):

$$
M_{m}^{(L)}=\sum_{\ell=1}^{L} \frac{k_{\ell} \tau_{\ell}}{\beta_{\ell}} \sum_{i=0}^{m}\left(\begin{array}{c}
m \\
i
\end{array}\right) \tau_{\ell}^{i} \overline{\bar{K}}^{p+m+1-i} \sum_{n=0}^{\bar{C}_{n}} \Phi_{\bar{T}}\left(\frac{p+m+2-n}{\beta_{\ell}}\right),
$$

where $\overline{\bar{K}}=-c /(p+m-i+1)$ and $\overline{\bar{C}}_{n}=\left(\begin{array}{c}p+m+1-i \\ n\end{array}\right) \bar{T}^{n}\left(-\tau_{\ell}\right)^{p+m+1-i-n}$.

Corollary 5 For $\dot{\gamma}(t)=c\left(t^{p-1}\left(1-t^{q}\right)\right) H(t-1)$, where $p, q$ are positive integers and $c$ is a positive constant, it follows from (25) and (12) that

$$
M_{m}^{(L)}=c \sum_{\ell=1}^{L} \frac{k_{\ell} \tau_{\ell}}{\beta_{\ell}} \sum_{i=0}^{m}\left(\begin{array}{c}
m \\
i
\end{array}\right) \tau_{\ell}^{i} \int_{0}^{\bar{T}} \Phi_{\overline{\bar{T}}}\left(\frac{i+1}{\beta_{\ell}}\right)\left(\tau^{p-1}-\tau^{p+q-1}\right) \tau^{m-i} d \tau,
$$

for $\bar{T}<1$, and

$$
M_{m}^{(L)}=c \sum_{\ell=1}^{L} \frac{k_{\ell} \tau_{\ell}}{\beta_{\ell}} \sum_{i=0}^{m}\left(\begin{array}{c}
m \\
i
\end{array}\right) \tau_{\ell}^{i} \int_{0}^{1} \Phi_{\hat{T}}\left(\frac{i+1}{\beta_{\ell}}\right)\left(\tau^{p-1}-\tau^{p+q-1}\right) \tau^{m-i} d \tau,
$$

for $\bar{T} \geq 1$.

\section{Analytic expression for stress for the single Kohlrausch $(L=1)$ when$$
\dot{\gamma}(t)=c t^{p-1}\left(1-t^{q}\right) H(t-1)
$$

Using equation (13) and Proposition 2, the analytic expression for stress for the Single Kohlrausch function $(L=1)$ when $\dot{\gamma}(t)=c\left(t^{p-1}\left(1-t^{q}\right)\right) H(t-1)$, 
is

$$
\sigma_{1}^{*}(t)=c \frac{k_{1} \tau_{1}}{\beta_{1}}\left[\sum_{i=0}^{p-1} \tilde{C}_{i}^{(1)} \Phi_{T\left(\beta_{1}, \tau_{1}\right)}\left(\frac{p-i}{\beta_{1}}\right)-\sum_{i=0}^{\hat{p}-1} \hat{C}_{i}^{(1)} \Phi_{T\left(\beta_{1}, \tau_{1}\right)}\left(\frac{\hat{p}-i}{\beta_{1}}\right)\right],
$$

for $0 \leq t<1$, and

$$
\sigma_{1}^{* *}(t)=c \frac{k_{1} \tau_{1}}{\beta_{1}}\left[\tilde{W}_{p}\left(\tau_{1}, \beta_{1}\right)-\hat{W}_{\hat{p}}\left(\tau_{1}, \beta_{1}\right)\right]
$$

for $t \geq 1$, where $\hat{p}=(p+q)$,

$$
\begin{aligned}
\tilde{C}_{i}^{(1)} & =\left(\begin{array}{c}
p-1 \\
i
\end{array}\right) t^{i}\left(-\tau_{1}\right)^{p-i-1} \\
\hat{C}_{i}^{(1)} & =\left(\begin{array}{c}
\hat{p}-1 \\
i
\end{array}\right) t^{i}\left(-\tau_{1}\right)^{\hat{p}-i-1}, \\
\tilde{W}_{p}\left(\tau_{1}, \beta_{1}\right) & =\sum_{i=0}^{p-1} \tilde{C}_{i}^{(1)} \int_{\left((t-1) / \tau_{1}\right)^{\beta_{1}}}^{\left(t / \tau_{1}\right)^{\beta_{1}}} \phi^{(p-i) / \beta_{1}-1} \exp (-\phi) d \phi \\
\hat{W}_{\hat{p}}\left(\tau_{1}, \beta_{1}\right) & =\sum_{i=0}^{\hat{p}-1} \hat{C}_{i}^{(1)} \int_{\left((t-1) / \tau_{1}\right)^{\beta_{1}}}^{\left(t / \tau_{1}\right)^{\beta_{1}}} \phi^{(\hat{p}-i) / \beta_{1}-1} \exp (-\phi) d \phi .
\end{aligned}
$$

The derivative of stress for the Single Kohlrausch function is

$$
\dot{\sigma}_{1}^{*}(t)=c k_{1}\left[t^{p-1}\left(1-t^{q}\right)-\left\{\tilde{J}_{p}\left(\tau_{1}, \beta_{1}\right)-\hat{J}_{\hat{p}}\left(\tau_{1}, \beta_{1}\right)\right\}\right] .
$$

where

$$
\begin{aligned}
& \tilde{J}_{p}\left(\tau_{1}, \beta_{1}\right)=\sum_{i=0}^{p-1} \tilde{C}_{i}^{(1)} \Phi_{T\left(\beta_{1}, \tau_{1}\right)}\left(\frac{p-i+\left(\beta_{1}-1\right)}{\beta_{1}}\right) \\
& \hat{J}_{\hat{p}\left(\tau_{1}, \beta_{1}\right)=} \sum_{i=0}^{\hat{p}-1} \hat{C}_{i}^{(1)} \Phi_{T\left(\beta_{1}, \tau_{1}\right)}\left(\frac{\hat{p}-i+\left(\beta_{1}-1\right)}{\beta_{1}}\right)
\end{aligned}
$$


for $0 \leq t<1$, and

$$
\dot{\sigma}_{1}^{* *}(t)=c k_{1}\left[t^{p-1}\left(1-t^{q}\right)-\left\{\bar{W}_{p}\left(\tau_{1}, \beta_{1}\right)-\overline{\bar{W}}_{\hat{p}}\left(\tau_{1}, \beta_{1}\right)\right\}\right] .
$$

where

$$
\begin{aligned}
& \bar{W}_{p}\left(\tau_{1}, \beta_{1}\right)=\sum_{i=0}^{p-1} \tilde{C}_{i}^{(1)} \int_{\left((t-1) / \tau_{1}\right)^{\beta_{1}}}^{\left(t / \tau_{1}\right)^{\beta_{1}}} \phi^{\left(p-i+\beta_{1}-1\right) / \beta_{1}-1} \exp (-\phi) d \phi, \\
& \bar{W}_{\hat{p}}\left(\tau_{1}, \beta_{1}\right)=\sum_{i=0}^{\hat{p}-1} \hat{C}_{i}^{(1)} \int_{\left((t-1) / \tau_{1}\right)^{\beta_{1}}}^{\left(t / \tau_{1}\right)^{\beta_{1}}} \phi^{\left(\hat{p}-i+\beta_{1}-1\right) / \beta_{1}-1} \exp (-\phi) d \phi,
\end{aligned}
$$

for $t \geq 1$.

\subsection{Recovery of Kohlrausch parameters: single Kohlrausch}

Husain and Anderssen [11] recently established theoretically how the unknown parameters (that is, $k_{\ell}, \beta_{\ell}, \tau_{\ell}, \ell=1,2, \ldots, L$ ) in such models can be recovered using moments of the measured stress, and of the corresponding known applied strain rates. For the single Kohlrausch situation where $L=1$, the algebraic relationships for the three moments of both the measured stress and the applied strain-rate can be rearranged to yield a strictly monotone formula for $\beta_{1}$, an explicit formula for $\tau_{1}$ in terms of $\beta_{1}$, and an explicit formula for $k_{1}$ in terms of $\tau_{1}$ and $\beta_{1}$. Together, they yield an existence and uniqueness proof for the parameters $\beta_{1}, \tau_{1}$ and $k_{1}$.

Here, the above results are used to propose some alternative methods. They exploit the fact that, for arbitrary $t$, analytic expressions $\sigma_{1}^{*}(t)$ and $\dot{\sigma}_{1}^{*}(t)$ are known functions involving the unknowns $k_{1}, \beta_{1}$ and $\tau_{1}$. Three possible alternatives follow. 
1. Choose a set of specific values of $t$ at which $\sigma_{1}^{*}(t)$ and $\dot{\sigma}_{1}^{*}(t)$ are evaluated and use the resulting algebraic expressions to construct algorithms for the estimation of $\beta_{1}, \tau_{1}$ and $k_{1}$. This is illustrated below.

2. For a large set of $t$ values on an appropriate even grid, construct the corresponding overdetermined system of equations, which is then solved using an appropriate non-linear least squares algorithm.

3. In order to construct interesting algebraic formulas which could, after appropriate algebraic manipulation, yield simple algorithms for the estimation of $\beta_{1}, \tau_{1}$ and $k_{1}$, utilise the algebraic expressions for $\sigma_{1}^{*}(t)$, $\dot{\sigma}_{1}^{*}(t)$, etc., for different choices for the above polynomial strain-rates (that is, for different values of $c, p$ and $q$ ).

To illustrate, examine the alternative 1 . Consider the situation where $L=1$, $p=2$ and $q=1$. Then equations (35) and (37) become

$$
\sigma_{1}^{*}(t)=c \frac{k_{1} \tau_{1}}{\beta_{1}}\left[P_{1}(t) \Phi_{T_{t}^{*}}\left(\frac{1}{\beta_{1}}\right)+\tau_{1} P_{2}(t) \Phi_{T_{t}^{*}}\left(\frac{2}{\beta_{1}}\right)-\tau_{1}^{2} \Phi_{T_{t}^{*}}\left(\frac{3}{\beta_{1}}\right)\right],
$$

and

$$
\begin{aligned}
\dot{\sigma}_{1}^{*}(t)=c k_{1}[ & P_{0}(t)-\left[P_{1}(t) \Phi_{T_{t}^{*}}(1)+\tau_{1} P_{2}(t) \Phi_{T_{t}^{*}}\left(\frac{1+\beta_{1}}{\beta_{1}}\right)\right. \\
& \left.\left.-\tau_{1}^{2} \Phi_{T_{t}^{*}}\left(\frac{2+\beta_{1}}{\beta_{1}}\right)\right]\right],
\end{aligned}
$$

where $T_{t}^{*}=T\left(\tau_{1}, \beta_{1}\right), P_{0}(t)=t(1-t), P_{1}(t)=\left(t-t^{2}\right)$ and $P_{2}(t)=(2 t-1)$.

These two equations involve the three unknowns $k_{1}, \beta_{1}$ and $\tau_{1}$. The aim then is to determine various specific values for $t$ such that the resulting equations can be combined to break the confounding involving $k_{1}, \beta_{1}$ and $\tau_{1}$.

For example, if one chooses $t=1 / 2$, then equations (39) and (40) become

$$
\sigma_{1}^{*}\left(\frac{1}{2}\right)=c \frac{k_{1} \tau_{1}}{\beta_{1}}\left[\frac{1}{4} \Phi_{T_{1 / 2}^{*}}\left(\frac{1}{\beta_{1}}\right)-\tau_{1}^{2} \Phi_{T_{1 / 2}^{*}}\left(\frac{3}{\beta_{1}}\right)\right],
$$




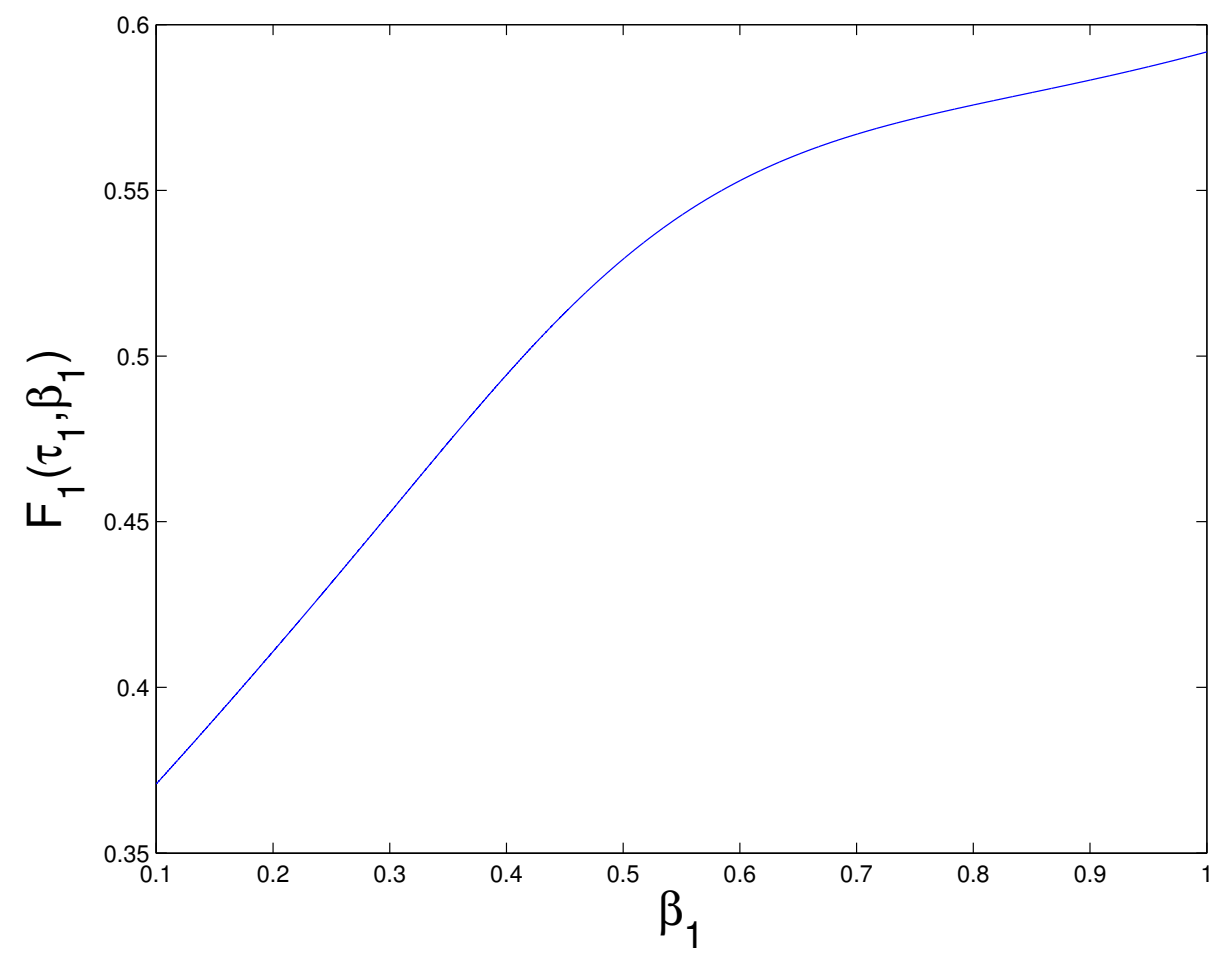

Figure 1: Plot of $F_{1}\left(\tau_{1}, \beta_{1}\right)$ as a function of $\beta_{1}$ with $t=0.5$ and $\tau_{1}=0.25$.

$$
\dot{\sigma}_{1}^{*}\left(\frac{1}{2}\right)=c k_{1}\left[\frac{1}{4}\left(1-\Phi_{T_{1 / 2}^{*}}(1)\right)+\tau_{1}^{2} \Phi_{T_{1 / 2}^{*}}\left(\frac{2+\beta_{1}}{\beta_{1}}\right)\right] .
$$

Dividing (41) by (42), one obtains

$$
F_{1}\left(\tau_{1}, \beta_{1}\right)=\frac{\frac{\tau_{1}}{4} \Phi_{T_{1 / 2}^{*}}\left(1 / \beta_{1}\right)-\tau_{1}^{3} \Phi_{T_{1 / 2}^{*}}\left(3 / \beta_{1}\right)}{\beta_{1}\left[\frac{1}{4}\left(1-\Phi_{T_{1 / 2}^{*}}(1)\right)+\tau_{1}^{2} \Phi_{T_{1 / 2}^{*}}\left(\frac{2+\beta_{1}}{\beta_{1}}\right)\right]}=A_{1}
$$

where $A_{1}=\sigma_{1}^{*}\left(\frac{1}{2}\right) / \dot{\sigma}_{1}^{*}\left(\frac{1}{2}\right)$ is known.

Using Proposition 1 and other algebraic identities (see Appendix A), it 
can be proved that, for fixed $\tau_{1}>0$, and $\frac{1}{\beta_{1}}>1, F_{1}\left(\tau_{1}, \beta_{1}\right)$ is a decreasing function of $\beta_{1}$. Figure 1 illustrates this monotonic decrease.

So, for a given $\tau_{1}$, one can estimate the parameter $\beta_{1}$. If necessary, one then uses this value for $\beta_{1}$ to improve the value of $\tau_{1}$ and continue the process iteratively until convergence. Equation (43) then determines $k_{1}$. Clearly, there are other ways in which equation (43) can be solved numerically to derive estimates of $\beta_{1}, \tau_{1}$ and $k_{1}$.

\section{A Monotonicity of $F_{1}\left(\tau_{1}, \beta_{1}\right)$}

Let

$$
F_{1}\left(\tau_{1}, \beta_{1}\right)=\frac{\tau_{1} \Phi_{T_{1 / 2}^{*}}\left(1 / \beta_{1}\right)-4 \tau_{1}^{3} \Phi_{T_{1 / 2}^{*}}\left(3 / \beta_{1}\right)}{\beta_{1}\left[1-\Phi_{T_{1 / 2}^{*}}(1)+4 \tau_{1}^{2} \Phi_{T_{1 / 2}^{*}}\left(\frac{2+\beta_{1}}{\beta_{1}}\right)\right]},
$$

where $F_{1}\left(\tau_{1}, \beta_{1}\right)=\sigma_{1}^{*}\left(\frac{1}{2}\right) / \dot{\sigma}_{1}^{*}\left(\frac{1}{2}\right)$ is known and $T_{1 / 2}^{*}=\left(1 / 2 \tau_{1}\right)^{\beta_{1}}$.

Let

$$
\begin{aligned}
& u=\tau_{1} \Phi_{T_{1 / 2}^{*}}\left(\frac{1}{\beta_{1}}\right)-4 \tau_{1}^{3} \Phi_{T_{1 / 2}^{*}}\left(\frac{3}{\beta_{1}}\right), \\
& v=\beta_{1}\left[1-\Phi_{T_{1 / 2}^{*}}(1)+4 \tau_{1}^{2} \Phi_{T_{1 / 2}^{*}}\left(\frac{2+\beta_{1}}{\beta_{1}}\right)\right] .
\end{aligned}
$$

Differentiating $u$ with respect to $T_{1 / 2}^{*}$ yields

$$
\frac{d u}{d T_{1 / 2}^{*}}=\tau_{1} \exp (-t) T_{1 / 2}^{* 1 / \beta_{1}-1}\left[1-4 \tau_{1}^{2} T_{1 / 2}^{* 2 / \beta_{1}}\right]
$$

Since $T_{1 / 2}^{* 2 / \beta_{1}}=1 / 4 \tau_{1}^{2}$, hence one obtains

$$
\frac{d u}{d T_{1 / 2}^{*}}=0
$$


Differentiating $v$ with respect to $T_{1 / 2}^{*}$ yields

$$
\frac{d v}{d T_{1 / 2}^{*}}=\frac{1}{T_{1 / 2}^{*} \log \left(T_{1 / 2}^{*}\right)}\left[1-\Phi_{T_{1 / 2}^{*}}(1)+4 \tau_{1}^{2} \Phi_{T_{1 / 2}^{*}}\left(\frac{2+\beta_{1}}{\beta_{1}}\right)\right] .
$$

Hence, differentiating $F_{1}\left(\tau_{1}, \beta_{1}\right)$ with respect to $T_{1 / 2}^{*}$, one obtains

$$
\frac{d F_{1}\left(\tau_{1}, \beta_{1}\right)}{d T_{1 / 2}^{*}}=-u \frac{d v / d T_{1 / 2}^{*}}{v^{2}}
$$

Proposition 6

$$
-1+\Phi_{T_{1 / 2}^{*}}(1)-4 \tau_{1}^{2} \Phi_{T_{1 / 2}^{*}}\left(\frac{2+\beta_{1}}{\beta_{1}}\right)<0 .
$$

Proof: Equation (51) can be written as

$$
-1+\Phi_{T_{1 / 2}^{*}}(1)-4 \tau_{1}^{2} \Phi_{T_{1 / 2}^{*}}\left(\frac{2+\beta_{1}}{\beta_{1}}\right)=-1+\Phi_{T_{1 / 2}^{*}}(1)\left(1-4 \tau_{1}^{2} \frac{\Phi_{T_{1 / 2}^{*}}\left(\frac{2+\beta_{1}}{\beta_{1}}\right)}{\Phi_{T_{1 / 2}^{*}}(1)}\right) .
$$

Since from Proposition 1, it can be shown that

$$
\frac{\Phi_{T_{1 / 2}^{*}}\left(\frac{2+\beta_{1}}{\beta_{1}}\right)}{T_{1 / 2}^{* \frac{2+1}{\beta_{1}}-1} \Phi_{T_{1 / 2}^{*}}(1)}=4 \tau_{1}^{2} \frac{\Phi_{T_{1 / 2}^{*}}\left(\frac{2+\beta_{1}}{\beta_{1}}\right)}{\Phi_{T_{1 / 2}^{*}}(1)}<1
$$

and

$$
\Phi_{T_{1 / 2}^{*}}(1)=1-\exp \left(-T_{1 / 2}^{*}\right)<1
$$

Hence, one obtain inequality (51).

Proposition 7

$$
u=\tau_{1} \Phi_{T_{1 / 2}^{*}}\left(\frac{1}{\beta_{1}}\right)-4 \tau_{1}^{3} \Phi_{T_{1 / 2}^{*}}\left(\frac{3}{\beta_{1}}\right)>0
$$


Proof: Equation (55) can be written as

$$
u=\tau_{1} \Phi_{T_{1 / 2}^{*}}\left(\frac{1}{\beta_{1}}\right)\left[1-4 \tau_{1}^{2} \frac{\Phi_{T_{1 / 2}^{*}}\left(3 / \beta_{1}\right)}{\Phi_{T_{1 / 2}^{*}}\left(1 / \beta_{1}\right)}\right] .
$$

To prove Proposition B, one exploits the Feng Qi Inequality given in the Subsection 2.3. For $s=3 / \beta_{1}$ and $r=1 / \beta_{1}, g(t)=t$, is an integrable positive function on the interval between 0 and $T_{1 / 2}^{*}$, because

$$
\int_{0}^{T_{1 / 2}^{*}} t d t=T_{1 / 2}^{* 2} / 2<\infty
$$

and, consequently, $\exp (t) g(t)=t \exp (t)$ is increasing. For this choice, the inequality yields

$$
\frac{\Phi_{T_{1 / 2}^{*}}\left(3 / \beta_{1}\right)}{\Phi_{T_{1 / 2}^{*}}\left(1 / \beta_{1}\right)} \leq\left(\frac{3+\beta_{1}}{1+\beta_{1}}\right) T_{1 / 2}^{* 2 / \beta_{1}}=\frac{1}{4 \tau_{1}^{2}}\left(1-\frac{2}{\beta_{1}+3}\right) .
$$

Since $0<\beta_{1}<1$, hence

$$
4 \tau_{1}^{2} \frac{\Phi_{T_{1 / 2}^{*}}\left(3 / \beta_{1}\right)}{\Phi_{T_{1 / 2}^{*}}\left(1 / \beta_{1}\right)} \leq 1 .
$$

This implies inequality (55).

Proposition $8 F_{1}\left(\tau_{1}, \beta_{1}\right)$ is either strictly increasing or decreasing function of $\beta_{1}$, depending on the fixed value of $\tau_{1}$.

Proof: Depending on the fixed value of $\tau_{1}$, and consequently the sign of $\log \left(T_{1 / 2}^{*}\right)$, from $(49)$

$$
\frac{d v}{d T_{1 / 2}^{*}} \begin{cases}>0, & \text { if } \log \left(T_{1 / 2}^{*}\right)>0 \\ <0, & \text { if } \log \left(T_{1 / 2}^{*}\right)<0\end{cases}
$$


Therefore, this implies

$$
\frac{d F_{1}\left(\tau_{1}, \beta_{1}\right)}{d T_{1 / 2}^{*}} \begin{cases}<0, & \text { if } \frac{d v}{d T_{1 / 2}^{*}}>0 \\ >0, & \text { if } \frac{d v}{d T_{1 / 2}^{*}}<0\end{cases}
$$

\section{References}

[1] M. Abramowitz and I. A. Stegun. Handbook of Mathematical Functions, Applied Mathematical Series. New York: Dover, 55:5, 1965. C940

[2] R. S. Anderssen and R. J. Loy. Completely Monotone Fading Memory Relaxation Modulii. Bull. Austral. Math. Soc., 65:449-460, 2002. C939

[3] R. S. Anderssen and R. J. Loy. Rheological Implications of Completely Monotone Fading Memory. Journal of Rheology, 46(6):1459-1472, 2002. C939

[4] R. S. Anderssen, Saiful A. Husain and R. J. Loy. Kohlrausch Functions: Properties and Applications, ANZIAM Journal, $\mathrm{E}(45): \mathrm{C} 800-\mathrm{C} 816,2004$. http://anziamj.austms.gov.au/V45/CTAC2003/Ande C939

[5] A. N. Beris and B. J. Edwards. On the Admissability Criteria for Linear Viscoelasticity Kernels. Rheol. Acta, 32:505-510, 1993. C939

[6] L. Boltzmann. Zur Theorie der Elastischen Nachwirkung. Ann. Phys. Chem., 7:624-657, 1876. C939

[7] A. R. Davies and R. S. Anderssen. Sampling Localization in Determining the Relaxation Spectrum. Journal of Non-Newtonian Fluid Mechanics, 73:163-179, 1997. C937 
[8] W. A. Day. On Monotonocity of the Relaxation Functions of Viscoelastic Materials. Proc. Camb. Philos. Soc., 67:503-508, 1970. C939

[9] W. Feller. An Introduction to Probability Theory and its Applications. John Wiley and Sons, New York, 2, 1970. C939

[10] A. Z. Grinshpan, M. E. H. Ismail, and D. L. Milligan. On Complete Monotonocity and Diesel Fuel Spray. Math. Intelligencer, 22:43-53, 2000. C939

[11] Saiful A. Husain and R. S. Anderssen. Modelling the Relaxation Modulus of Linear Viscoelasticity Using Kohlrausch Functions. J. Non-Newtonian Fluid Mechanics, 125:159-170, 2005. C937, C948

[12] Feng Qi. Monotonicity Results and Inequalities For the Gamma and Incomplete Gamma Functions. Mathematical Inequalities and Applications, 5(1):61-67, 2002.

http://www.mia-journal.com/main.sap C940

[13] R. Kohlrausch. Theorie des Elektrischen Rückstandes in der Leidener Flasche. Pogg. Ann. Phys. Chem., 91:179-214, 1854. C939

[14] D. V. Widder. The Laplace Transform. Princeton University Press, Princeton, 1946. C939

[15] G. Williams and David C. Watts. Non-symmetrical Dielectric Relaxation Behaviour Arising from a Simple Empirical Decay Function. Trans. Faraday Soc., 66:80-85, 1970. C939

[16] G. Williams. The Non-crystalline Higher Polymer and the Related Material lies between the Electricity Relaxes the Characteristic. IEEE Trans on Electrical Insulation, EI-20(5):843-857, 1985. C939 